\title{
Amphioxus and lamprey AP-2 genes: implications for neural crest evolution and migration patterns
}

\author{
Daniel Meulemans and Marianne Bronner-Fraser* \\ Division of Biology, 139-74, California Institute of Technology, Pasadena, CA 91125, USA \\ *Author for correspondence (e-mail: mbronner@caltech.edu)
}

Accepted 30 July 2002

\section{SUMMARY}

The neural crest is a uniquely vertebrate cell type present in the most basal vertebrates, but not in cephalochordates. We have studied differences in regulation of the neural crest marker AP-2 across two evolutionary transitions: invertebrate to vertebrate, and agnathan to gnathostome. Isolation and comparison of amphioxus, lamprey and axolotl AP-2 reveals its extensive expansion in the vertebrate dorsal neural tube and pharyngeal arches, implying co-option of AP-2 genes by neural crest cells early in vertebrate evolution. Expression in non-neural ectoderm is a conserved feature in amphioxus and vertebrates, suggesting an ancient role for AP-2 genes in this tissue. There is also common expression in subsets of ventrolateral neurons in the anterior neural tube, consistent with a primitive role in brain development. Comparison of AP-2 expression in axolotl and lamprey suggests an elaboration of cranial neural crest patterning in gnathostomes. However, migration of AP-2-expressing neural crest cells medial to the pharyngeal arch mesoderm appears to be a primitive feature retained in all vertebrates. Because AP-2 has essential roles in cranial neural crest differentiation and proliferation, the co-option of AP-2 by neural crest cells in the vertebrate lineage was a potentially crucial event in vertebrate evolution.

Key words: AP-2, Amphioxus, Lamprey, Neural crest, Migration

\section{INTRODUCTION}

A defining event in vertebrate evolution was the appearance of neural crest cells (Gans and Northcutt, 1983). These cells form most of the structures that distinguish vertebrates from other chordates, including the skeletomusculature of the cranium, face, jaws and pharynx, and most of the peripheral nervous system. Despite their importance, little is known about the evolutionary origins of neural crest cells.

In the most basal vertebrate studied, lamprey, an essentially modern neural crest generates almost all of the derivatives seen in gnathostomes (Langille and Hall, 1988). By contrast, the most vertebrate-like invertebrates, the cephalochordates, appear to lack even a rudimentary neural crest. Furthermore, the fossil record offers no obvious intermediate forms that display features suggestive of a primitive neural crest.

Within the gnathostomes, the molecular mechanisms that underlie neural crest induction are largely conserved (for a review, see LaBonne and Bronner-Fraser, 1999). Input from BMP, Wnt and FGF signaling pathways activate a complement of transcription factors at the neural plate border, including Snail, Twist, Zic, Id, AP-2, FoxD3, Distal-less, Msx and Pax genes. A subset of these factors has been shown to cross- and autoregulate, such that a rough outline of their regulatory relationships is emerging (Sasai et al., 2001).

Amphioxus and lamprey are useful organisms for investigating neural crest evolution as they both diverged near the time neural crest first appeared. Amphioxus, a cephalochordate, separated from the vertebrate lineage before the origin of neural crest and is thought to approximate the ancestral pre-vertebrate chordate. Expression studies in amphioxus reveal that some of the genetic machinery needed to create neural crest cells (including BMP-4, Snail, Pax-3, Wnt7B, Distalless and Msx) was in place before bona fide neural crest cells appeared (reviewed by Holland and Holland, 2001). Lamprey diverged from other vertebrates relatively soon after the neural crest arose and is thought to display primitive features lost or masked in gnathostomes.

In this study, we focus on the regulatory evolution of the transcriptional activator AP-2 as a starting point for dissecting the molecular history of neural crest cells. AP-2 is a robust neural crest marker shown to be essential for cranial neural crest development in vertebrates. The vertebrate AP-2 family consists of four genes (AP- $2 \alpha, \beta, \gamma$ and $\delta$ ) that have dynamic and largely overlapping patterns of expression during embryogenesis (reviewed by Hilger-Eversheim et al., 2000) [for description of AP-2 delta see Zhao et al. (Zhao et al., 2001)]. At gastrula stages, AP-2 transcripts are initially observed in non-neural ectoderm. As neurulation proceeds, AP-2 expression is extinguished in non-neural ectoderm and upregulated in the neural folds, marking neural crest cells before, during and after their migration. AP- $2 \alpha$ is functionally important for neural crest cells, as null mice almost completely lack cranial neural crest derivatives (Schorle et al., 1996; Zhang 
et al., 1996). In addition, AP-2 is necessary for expression of HoxA2 in the neural crest, indicating an indirect role for AP2 genes in neural crest patterning (Maconochie et al., 1999).

We describe the isolation of amphioxus and lamprey AP-2 homologs and compare their expression patterns with that of AP-2 in the gnathostome axolotl. Using this broad comparative base, we span two important evolutionary transitions: the divergence of vertebrates from invertebrates and the divergence of jawed vertebrates from agnathans. Across each transition, we observe differences in the deployment of AP-2 genes that are suggestive of key genetic and developmental changes during early vertebrate evolution. Taken together, our observations suggest a crucial role for AP-2 during neural crest evolution.

\section{MATERIALS AND METHODS}

\section{Embryo collection}

Adult amphioxus (Branchiostoma floridae) were collected from Old Tampa Bay, Florida and electrostimulated to induce gamete release. Eggs were fertilized and embryos cultured, staged and fixed as described by Holland et al. (Holland et al., 1996b). Embryos of the lamprey Petromyzon marinus were collected, staged and fixed at the Lake Huron biological station according to the methods of Tomsa and Langeland (Tomsa and Langeland, 1999) and provided as a gift by David McCauley. Albino embryos of the Mexican axolotl, (Ambystoma mexicanum) were obtained from the axolotl colony in Bloomington, Indiana and raised and staged as described by Epperlein et al. (Epperlein et al., 2000).

\section{AP-2 gene isolation}

Amphioxus and lamprey embryonic cDNA libraries were the generous gifts of Jim Langeland. 500 and $200 \mathrm{bp}$ fragments of amphioxus and lamprey AP-2 genes, respectively, were amplified directly from diluted lambda phage libraries by degenerate PCR with the following primers: for amphioxus, 5' primer GTRTTCTGYKCAGKYCCYGGICG and 3' primer GWKATVAGGKWGAAGTGSGTCA; for lamprey, 5' primer CCVCCIGARTGCCTSAAYGC and 3 ' primer GAAGTCICGVGCSARRTG. Amplified fragments were used to screen the libraries at high stringency (final wash $0.2 \times \mathrm{SSC}$ ) to isolate full-length clones. Phagemids were excised and inserts sequenced completely from both ends. Low stringency screens of the amphioxus cDNA library and an arrayed amphioxus genomic library were performed as described for Southern blot analysis.

\section{Phylogenetic analysis}

The conceptual protein products of the amphioxus and lamprey AP2 transcripts were aligned with vertebrate and Drosophila AP-2 protein sequences. Axolotl AP-2 was not used for analysis as only a partial sequence is available. A phylogenetic tree was created within the ClustalX program (Thompson et al., 1997) using the neighborjoining method of Saitou and Nei (Saitou and Nei, 1987). Bootstrap values were determined by 1000 resamplings of alignment data. GenBank Accession Numbers for the aligned sequences are: mouse AP-2 $\alpha$, NP035677, mouse AP-2 $\beta$, Q61313, mouse AP-2 $\gamma$, Q61312, mouse AP-2 $\delta$, AAL16940, chicken AP-2 $\alpha$, AAB65081, chicken AP$2 \beta$, AAC26111, human AP-2 $\alpha$, NP003211, human AP-2 $\beta$, NP003212, human AP-2 $\gamma$, XP009543, Xenopus AP-2 $\alpha$, S34449, Drosophila AP-2, CAA07279.

\section{Hox2 in silico cis regulatory analysis}

Genomic sequences surrounding the transcriptional start of Drosophila proboscipedia and AmphiHox2 were scanned for consensus AP-2 binding sites using the MatInspector v2.2 program. Core and matrix similarities were set at the default values of 0.75 and 0.85, respectively. Accession Numbers are NG000110 for proboscipedia and $\mathrm{AB} 050888$ and $\mathrm{AB} 050887$ for AmphiHox2 genomic sequences.

\section{In situ hybridization}

In situ hybridization on amphioxus embryos was performed as described by Holland (Holland, 1996a) with the omission of deacetylation and RNAse treatments. In addition, post-hybridization washes were in PBS-Tween $0.1 \%$, rather than SSC, and the blocking solution was $2 \mathrm{mg} / \mathrm{ml} \mathrm{BSA} / 2 \%$ sheep serum in PBS-Tween $0.1 \%$. Riboprobes against the DNA binding/dimerization domain and fulllength cDNA yielded identical staining patterns.

In situ hybridization on axolotl and lamprey embryos were as described by Henrique et al. (Henrique et al., 1995) with the addition of an extra 12 hour wash in MAB-Tween. Tween- 20 concentrations for $\mathrm{PBS}$ and $\mathrm{MAB}$ solutions were increased to $0.2 \%$. Proteinase $\mathrm{K}$ treatments were also adjusted to $50 \mu \mathrm{g} / \mathrm{ml}$ for 15 minutes for lamprey embryos and $10 \mu \mathrm{g} / \mathrm{ml}$ for 4 minutes for axolotl embryos. Hybridization was at $65^{\circ} \mathrm{C}$. For lamprey, the riboprobe was generated against a 500 bp region of the DNA binding/dimerization domain. The axolotl AP-2 riboprobe was prepared as previously described (Epperlein et al., 2000).

\section{Southern blot analysis}

Genomic DNA from five adult amphioxus was purified and digested with four restriction enzymes (ApaI, ClaI, EcoRV and HindIII). Genomic DNA from a single adult lamprey was isolated and digested with six restriction enzymes (ApaI, EcoRI, HindIII, NcoI, PstI and $S t u I)$. Digests were electrophoresed on $0.7 \%$ agarose gels and blotted onto GeneScreen Plus filters (NEN Life Science Products). Homologous $200 \mathrm{bp}$ probes were designed to intra-exonic regions of the DNA binding and dimerization domains of the amphioxus and lamprey AP-2 genes. Intron-exon boundaries were deduced from human AP-2 $\alpha$ genomic sequences (Bauer et al., 1994) and partial sequencing of amphioxus AP-2 cosmids. Southern blots were hybridized in $6 \times \mathrm{SSC} / 5 \% \mathrm{SDS} / 100 \mu \mathrm{g} / \mathrm{ml}$ sheared herring sperm DNA $/ 5 \times$ Denhardt's solution at $60^{\circ} \mathrm{C}$ to ${ }^{32} \mathrm{P}$-labeled probes. Washes were in $2 \times \mathrm{SSC}, 0.5 \% \mathrm{SDS}$ at $55^{\circ} \mathrm{C}$.

\section{Plastic sectioning}

Embryos were dehydrated in ethanol and embedded in Epon-Araldite. After polymerization for 72 hours at $60^{\circ} \mathrm{C}$, the embryos were sectioned to $10-15 \mu \mathrm{m}$ using a glass knife, coverslipped in Gelmount and photographed.

\section{RESULTS}

\section{Isolation of AP-2 from amphioxus and lamprey}

We used degenerate PCR to isolate AP-2 gene fragments from amphioxus and lamprey embryonic cDNA libraries. The fragments were then used to screen the libraries at high stringency for full-length clones. The longest cDNAs from each screen were completely sequenced. Within both cDNAs, open reading frames encoding proteins of exactly 498 amino acids were identified (Fig. 1A). The amphioxus and lamprey proteins were found to be 49 and 54 percent identical, respectively, to mouse AP-2 $\alpha$, with 74 and $78 \%$ identity over the DNA binding and dimerization domains. A region of high sequence similarity was also seen in the proline-rich transactivation domain. An arrayed amphioxus cosmid library was screened at low stringency, and four hybridizing cosmids were partially sequenced. 
A

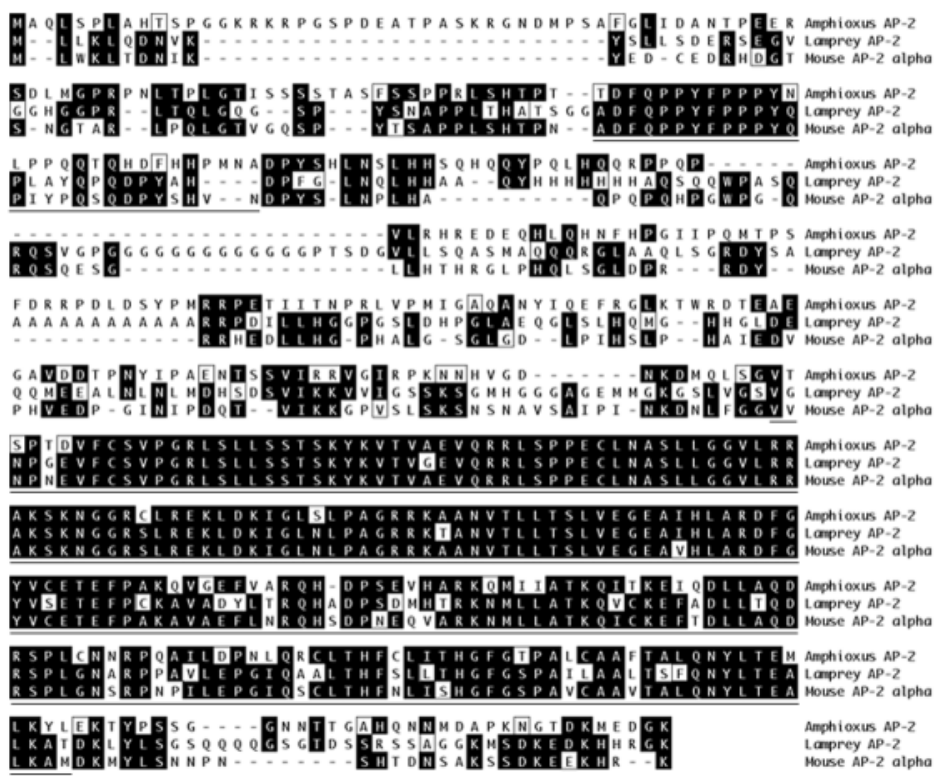

B

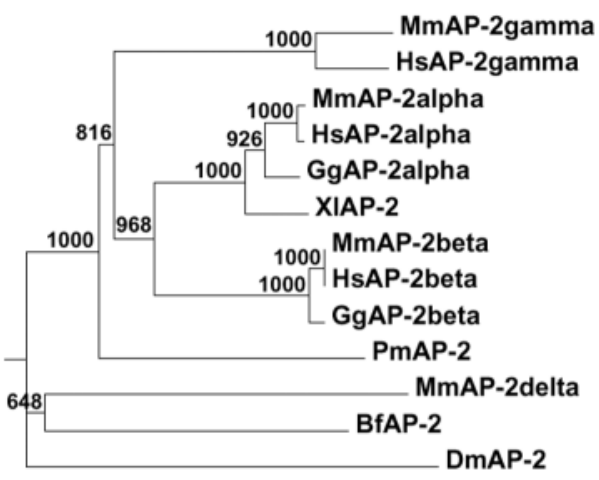

0.1

Fig. 1. Sequence analysis of amphioxus and lamprey AP-2 proteins. (A) Clustal alignment of amphioxus AP-2, lamprey AP-2 and mouse AP$2 \alpha$. Identical residues are shaded black, biochemically similar residues are boxed. Underlined regions of mouse AP-2 $\alpha$ represent the prolinerich transactivation domain (N-terminal) and the DNA-binding/dimerization domain (C-terminal). The regions of highest homology between the three sequences are within these functionally important domains. (B) Neighbor-joining phylogenetic tree of vertebrate and amphioxus AP-2 protein sequences. Drosophila serves as an outgroup. Numbers at branch points are confidence values derived from 1000 bootstrap resamplings of the alignment data. Sequence distance is indicated to the bottom left as substitutions per base. Lamprey AP-2 (Pm) is an outgroup to the gnathostome AP-2s and has no affinity for any one AP-2 family member, consistent with there being a single lamprey AP-2 gene. Amphioxus AP-2 (Bf) falls outside the vertebrate clade. The divergent mouse AP- $2 \delta$ groups with amphioxus AP- 2 at low bootstrap values, and its phylogenetic relationship to the other vertebrate AP-2 family members is unclear. Dm, Drosophila melanogaster; $\mathrm{Bf}$, Branchiostoma floridae; Pm, Petromyzon marinus; Hs, Homo sapiens; Mm, Mus Musculus; Gg, Gallus gallus; Xl, Xenopus laevis.

\section{Southern blot analysis}

Low-stringency Southern blot analysis was used to estimate the number of AP-2 genes in the amphioxus and lamprey genomes. In both cases, probes were created that recognized part of the highly conserved DNA-binding domain, but were likely to be intra-exonic based upon the genomic structure of human and amphioxus AP-2 genes.

Probing of genomic DNA from a single adult amphioxus revealed two strongly hybridizing fragments when digested with seven out of eight enzymes (data not shown). This raised the possibility that there was more than one AP-2 family member in the amphioxus genome. To test this, we re-probed the cDNA library at low stringency and detected no additional AP-2 cDNAs. Low stringency screening of an arrayed amphioxus genomic library also yielded no new AP-2 gene sequences. We then investigated whether the multiple fragments were due to polymorphism at the AP-2 locus. Genomic DNA from five individual adult amphioxus were digested with four enzymes. All five adults had different restriction fragment length profiles (Fig. 2A). For each enzyme, two to four different fragments were observed in total, with each animal possessing only one or two fragment types per enzyme. Collectively, the results are consistent with various homo- and heterozygotic combinations of several restriction fragment length alleles at a single highly polymorphic locus. Based upon this, and the fact that low-stringency screens of cDNA and genomic libraries consistently yielded a single gene, we conclude that there is a single AP-2 gene in the amphioxus genome.

Low-stringency Southern blot of genomic DNA from an individual adult lamprey showed a single band in four out of five digests. Probing of lamprey genomic DNA with an amphioxus AP-2 probe yielded no discernable signal above background (data not shown).

\section{AP-2 gene phylogeny}

Amphioxus, lamprey, mouse, chicken, frog, human and Drosophila AP-2 sequences were aligned, and a phylogenetic tree was generated using the neighbor joining method (Fig. 1B). Axolotl AP-2 was not used for analysis as only a partial sequence is available. The deduced phylogeny shows amphioxus AP-2 falling outside of the vertebrate AP-2 clade, which includes lamprey AP-2 and gnathostome AP- $2 \alpha, \beta$ and $\gamma$. Within the vertebrate clade, lamprey AP-2 fails to group with any one gnathostome AP-2 isoform. This general topology is maintained when the DNA binding/dimerization domain alone is used for alignment.

Unexpectedly, the recently described mouse AP- $2 \delta$ fails to group with vertebrate AP-2 proteins when full-length sequences are aligned. When only the conserved DNA binding/dimerization domains are used for alignment, AP-2 $\delta$ also falls outside of the amphioxus/vertebrate clade (data not shown). Both phylogenetic positions are poorly supported by low bootstrap values and may reflect rapid evolution of AP- $2 \delta$ 


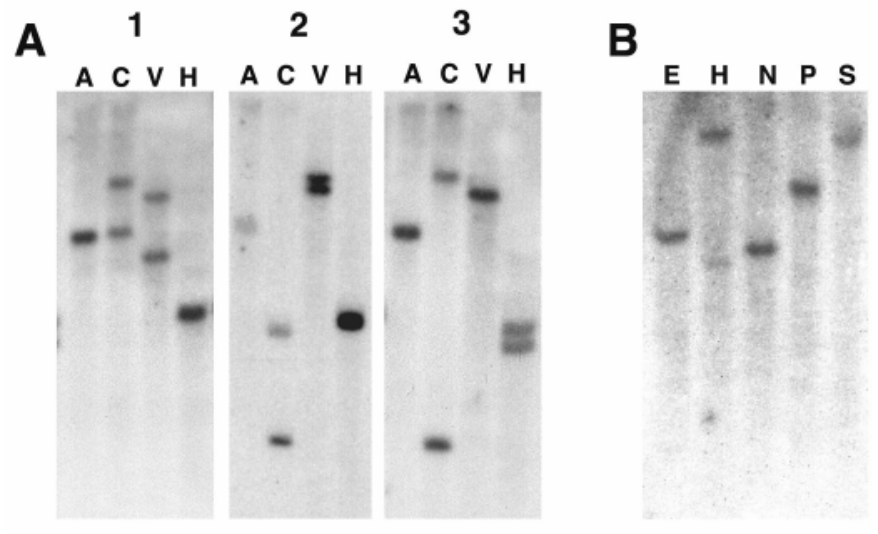

Fig. 2. Reduced stringency Southern blot analysis to estimate AP-2 gene number in the amphioxus and lamprey genomes. (A) Genomic DNA from five individual adult amphioxus was digested with four restriction enzymes and analyzed on the same Southern blot. Lanes from three representative individuals are shown. Interestingly, each animal gives a different banding pattern. For a particular enzyme, an individual has no more than two band types, and most of these bands are shared between individuals. For example, individuals 2 and 3 share lowest ClaI band, while all three individuals share a single ApaI band. Overall, the banding patterns are most consistent with various hetero- and homozygotic combinations of restriction fragment length polymorphisms at a single locus, suggesting there is one amphioxus AP-2 gene. (B) Genomic DNA from a single adult lamprey digested with five restriction enzymes. Four out of five digests yield a single band, consistent with there being a single lamprey AP-2 gene. A, ApaI; C, ClaI; V, EcoRV; H, HindIII; E, EcoRI; N, NcoI; P, PstI; S, StuI.

in gnathostomes or early divergence of AP- $2 \delta$ in the vertebrate lineage.

\section{Pattern of AP-2 expression in amphioxus}

Amphioxus development proceeds in a simplified vertebratelike manner, with the neural plate forming from dorsal ectoderm at 8-9 hours post-fertilization. In 9 hour neurulae, AP-2 transcripts are detected throughout the non-neural ectoderm (Fig. 3A,B). No expression is seen in the open neural plate or mesendoderm. After the onset of somitogenesis at 9.510 hours, non-neural ectoderm begins closing over the invaginating neural plate. In 11.5 hour neurulae, AP-2expressing ectoderm cells appear to be migrating over the closing neural plate (Fig. 3C,D,G). Upon hatching at 12 hours, the neurula is covered in ciliated AP-2-positive epidermis. Neurulation is completed under the epidermis by hour 18 . During this period, AP-2 ectodermal expression begins to recede from the anterior- and posterior-most ends of the larva (Fig. 3E). At 20 hours, a small spot of staining appears in the anterior gut, probably presaging formation of the left gut diverticulum. At 24 hours, this expression sharpens, marking the endodermal portion of the developing preoral pit (Fig. $3 \mathrm{~F}, \mathrm{H})$. Simultaneously, strong staining appears in the ventrolateral walls of the cerebral vesicle and expression in the epidermis fades (Fig. 3H,I,L). At 36 hours, the embryo has elongated to roughly twice its 18-hour length, and the mouth and first gill slit begin to form (Fig. 3K). Both the cerebral vesicle and pre-oral pit staining become markedly reduced after this time (Fig. 3J), but persist weakly until 4 days.

\section{Pattern of AP-2 expression in lamprey}

At 4 days, the neural plate of the lamprey embryo is a flattened area of dorsal ectoderm. At this stage, AP-2 staining is observed in non-neural ectoderm (Fig. 4A,F). As the neural plate condenses towards the dorsal midline around day 5, AP2 transcripts are detected at the edges of the neural plate and broadly in the adjacent ectoderm (data not shown). AP-2 is downregulated in the non-neural ectoderm at ventral and lateral levels.

At six days, AP-2 is expressed solely in the dorsal neural rod (Fig. 4C,G), forming a stripe that is disrupted anteriorly by a gap in expression near the protruding head (Fig. 4B). Although expression in non-neural ectoderm is extinguished in 6-day-old embryos, a new phase of epidermal expression begins at 7 days in the head (Fig. 4D). Scattered AP-2-positive cells appear throughout the head ectoderm, but are conspicuously absent from the otic placode. Also at 7 days, the anterior gap in neural rod expression sharpens, and sections reveal staining in surrounding head mesenchyme highly reminiscent of early migrating neural crest in other vertebrates (Fig. 4H).

At 7.5 days, separations in the head staining become discernable, suggestive of neural crest-free spaces between AP-2 positive streams (Fig. 4E). Horizontal sections reveal the initial outpocketing of first arch endoderm at this time with AP2 transcripts in the mesenchyme and dorsal neural tube (Fig. 4P). Sections at the level of the otic vesicle show accumulation of AP-2 signal in the space dorsal to the vesicle as well as in the mesenchyme below it, but never medial to the otic vesicle. (Fig. 4I)

At 8 days, divisions in the head staining become more obvious, and three broad areas of AP-2 expression can be distinguished - an anterior band and two more caudal swathes straddling the putative otic placode (Fig. 4K). Ventrally, in the region of the nascent pharyngeal arches, the two posterior streams fuse into one continuous mass, while the anterior stream splits into three smaller streams. The rostral-most stream sits just anterior to the area of the optic cup and probably represents the ophthalmic neural crest stream (Fig. $6 \mathrm{~B})$. Around the mouth, the rest of the anterior stream forks, marking cells in the mandibular arch and maxillary (anterior lip) region. Horizontal sections at 8.5 days show formation of the first three pharyngeal arches with AP-2 transcripts detected in the ectoderm, superficial to the ectoderm, and adjacent to pharyngeal endoderm (Fig. 4Q). From 8.5-9.0 days, staining in the area of the pharyngeal arches accumulates (Fig. 4L,M). In the trunk, staining in the dorsal neural tube and dorsal fin, and weak staining between the somites are apparent (data not shown).

At 10-11 days, a new phase of AP-2 neural expression begins in a subset of cells in the anterior neural tube (Fig. 4O). From days 11 to 12, gaps appear in the AP-2-positive arch mesenchyme where the pharyngeal endoderm and ectoderm meet to create the pharyngeal slits (Fig. 4N). Horizontal sections at 12 days show the formed arches, with AP-2 signal present medial and lateral to the pharyngeal mesoderm (Fig. 4R).

\section{Pattern of AP-2 expression in axolotl}

To facilitate comparison of lamprey and amphioxus AP-2 gene usage with that of gnathostomes, a developmental series of 

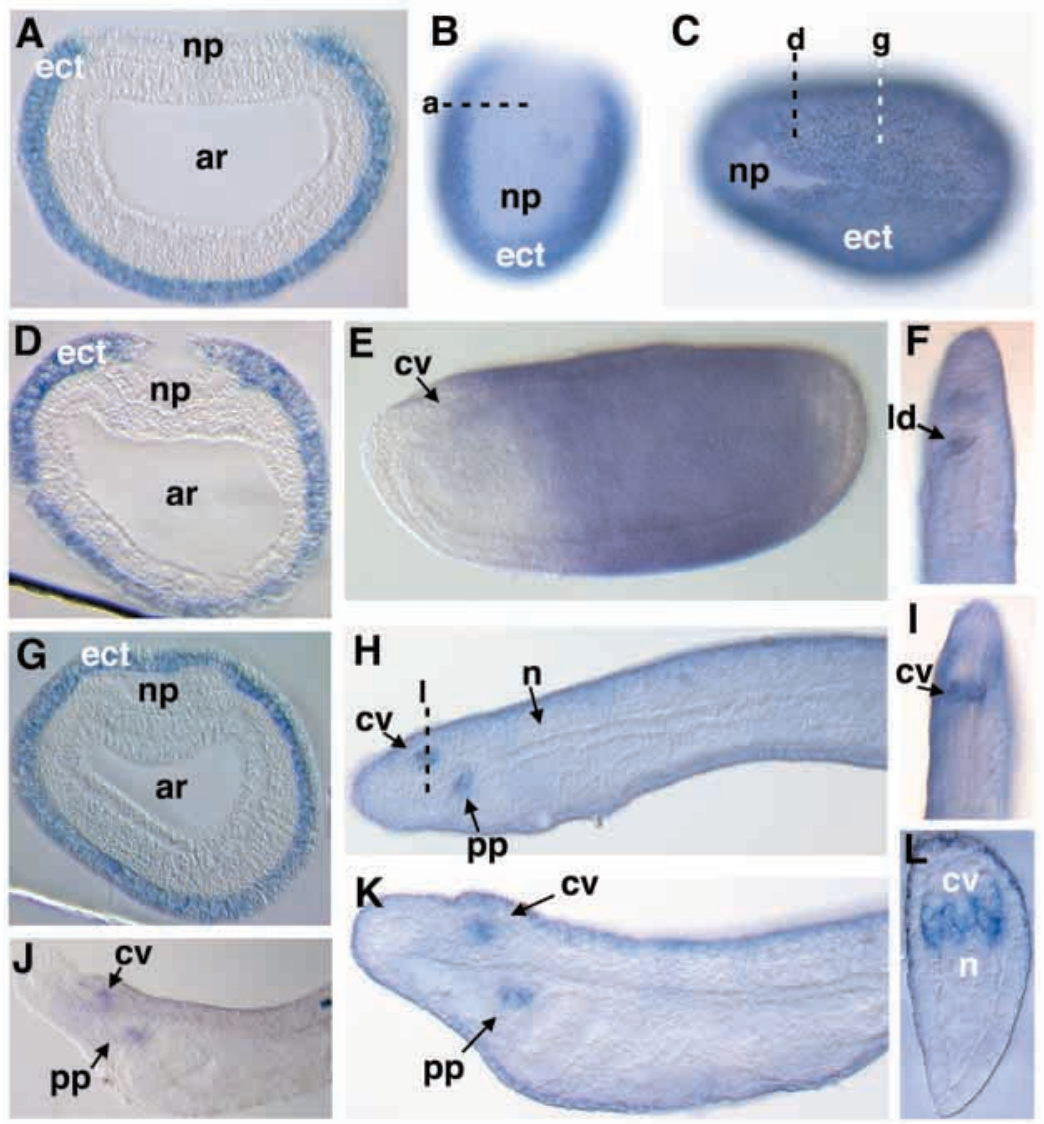

Fig. 3. AP-2 expression in amphioxus. (A) Cross-section through the 9 hour neurula depicted in $\mathrm{B}$ at position a, showing AP-2 transcripts in the non-neural ectoderm.(B) Dorsal anterior view of 9 hour neurula showing exclusion of AP-2 from the neural plate.

(C) Dorsal view of 11.5 hour neurula, anterior is towards the left. AP-2 is expressed in the epidermis overgrowing the neural plate. (D) Cross-section through the embryo in $\mathrm{C}$ at position d. (E) Side view of 18 hour neurula. AP-2 expression in the epidermis is being extinguished from the anterior- and posterior-most ends of the embryo. (F) Optical horizontal section of 24 hour embryo through the gut with anterior towards the top. A spot of AP-2 positive cells is apparent in the left gut diverticulum, the endodermal portion of the pre-oral pit (arrow). (G) Crosssection of 9 hour neurula in $\mathrm{C}$ at position $\mathrm{g}$. At more posterior levels, the AP-2 positive epidermis has completely covered the open neural plate. (H) Side view of 24 hour embryo with anterior to the left. AP-2 expression is seen in the cerebral vesicle and pre-oral pit, but has largely faded from the epidermis. (I) Optical horizontal section of 24 hour embryo in $\mathrm{H}$ at the level of the neural tube showing symmetrical AP-2 staining in the cerebral vesicle. Anterior is towards top. (J) Two-day larva with AP-2 expression persisting in the cerebral vesicle and pre-oral pit. (K) 36 hour larva with AP-2 expression in cerebral vesicle and pre-oral pit. (L) Crosssection through 24 hour embryo in $\mathrm{H}$ at position 1 . AP-2 expression in the cerebral vesicle is limited to ventrolateral levels. ar, archenteron; ect, non-neural ectoderm/epidermis; $\mathrm{np}$, neural plate; $\mathrm{n}$, notochord; $\mathrm{cv}$, cerebral vesicle; pp, pre-oral pit; ld, left gut diverticulum. axolotl embryos was probed for AP-2 transcripts. AP-2 expression in the axolotl has been described for stages just preceding and following neural crest migration and was found to mirror that of mouse, chicken and frog (Epperlein et al., 2000). Staining patterns in early neurulae, however, have not been previously described. At open neural plate stages, AP-2 transcripts are detected in the non-neural ectoderm, and are strongly expressed at the neural plate border (Fig. 5A, far right panel). As neurulation proceeds, AP-2 is further upregulated in the protruding neural folds and downregulated in the nonneural ectoderm. Upon neural tube closure, AP-2 staining in the dorsal aspect of the neural tube is maximal, while nonneural ectoderm has only a residual AP-2-positive signal (data not shown). Little or no staining is apparent in non-neural ectoderm at later stages.

\section{DISCUSSION}

In this study, we have used the expression of chordate AP-2 genes in two ways: (1) to suggest homology between structures where homology is well-supported by anatomy; and (2) as evidence of the regulatory history of AP-2 in the chordate lineage.

\section{Chordate AP-2 genes}

Single representatives of the AP-2 gene family were isolated from amphioxus and lamprey embryonic cDNA libraries. The presence of a single AP-2 gene in each genome was suggested by low-stringency genomic Southern blotting and phylogenetic analyses. This follows with gene numbers in amphioxus where a 1:3 or 1:4 correspondence of amphioxus to gnathostome gene homologs is usually observed, and supports the proposed scheme of two whole or partial genome duplications in the vertebrate lineage (for a review, see Holland, 1999). Limited data from lamprey indicate a homolog ratio closer to 1:2 when taking into account lamprey-specific gene duplication events (Sharman and Holland, 1998; Ueki et al., 1998; Ogasawara et al., 2000; Myojin et al., 2001; Neidert et al., 2001; Force et al., 2002). Thus, there is a chance that another lamprey AP-2 gene exists that was not detected. Furthermore, phylogenetic analysis leaves open the possibility that lamprey has an AP- $2 \delta$, as lamprey AP-2 groups with gnathostome $\alpha, \beta$ and $\gamma$, but not $\mathrm{AP}-2 \delta$. Whether this is due to rapid evolution of AP- $2 \delta$ in mammals or early divergence of AP- $2 \delta$ in vertebrates is unclear. Alternately, a second lamprey AP-2 may have been lost during evolution or double duplication of an ancestral AP2 gene occurred after the divergence of agnathans. Outside of phylum chordata, it is likely that having one AP-2 gene is the primitive condition for bilateria, as only a single AP-2 gene is found in Drosophila (Bauer et al., 1998; Monge and Mitchell, 1998). Overall, our data are consistent with the vertebrate genome double-duplication hypothesis, but are inconclusive as to the timing of these duplications relative to gnathostome origins.

\section{Early non-neural ectoderm AP-2 expression is ancestral}

A striking feature of AP-2 expression common to amphioxus, lamprey and axolotl, is robust expression in non-neural 
Fig. 4. AP-2 expression in lamprey. (A) Dorsal anterior view of 4-dayold neurula. AP-2 expression is observed in the non-neural ectoderm at the exclusion of the neural plate. (B) Dorsal anterior view of 6 day neurula showing expression confined to the neural rod. An AP-2-free gap disrupts the neural rod signal anteriorly. (C) Side view of embryo in $\mathrm{B}$ with head protruding upwards. Dorsal neural rod expression is indicated by the arrow. (D) Side view of 7-day-old embryo, anterior is towards the right. AP-2 expression is enhanced in the head epidermis and mesenchyme, but is excluded from the otic cup. (E) 7.5-day-old embryo showing AP-2 expression throughout the head. (F) Crosssection through the neurula in $\mathrm{A}$ at $\mathrm{f}$. AP-2 transcripts are absent from the neural plate. $(\mathrm{G})$ Cross-section through 6-day-old neurula in $\mathrm{C}$ at position g, showing AP-2 expression in the dorsal neural tube (arrow).

(H) Cross-section through 7-day-old embryo in $\mathrm{D}$ at position $\mathrm{h}$, showing AP-2 staining in dorsal neural tube, head mesenchyme and epidermis (arrows). (I) Cross-section of 7.5day-old embryo in $\mathrm{E}$ through the otic vesicle at position i. AP-2 transcripts are detected in the dorsal neural tube
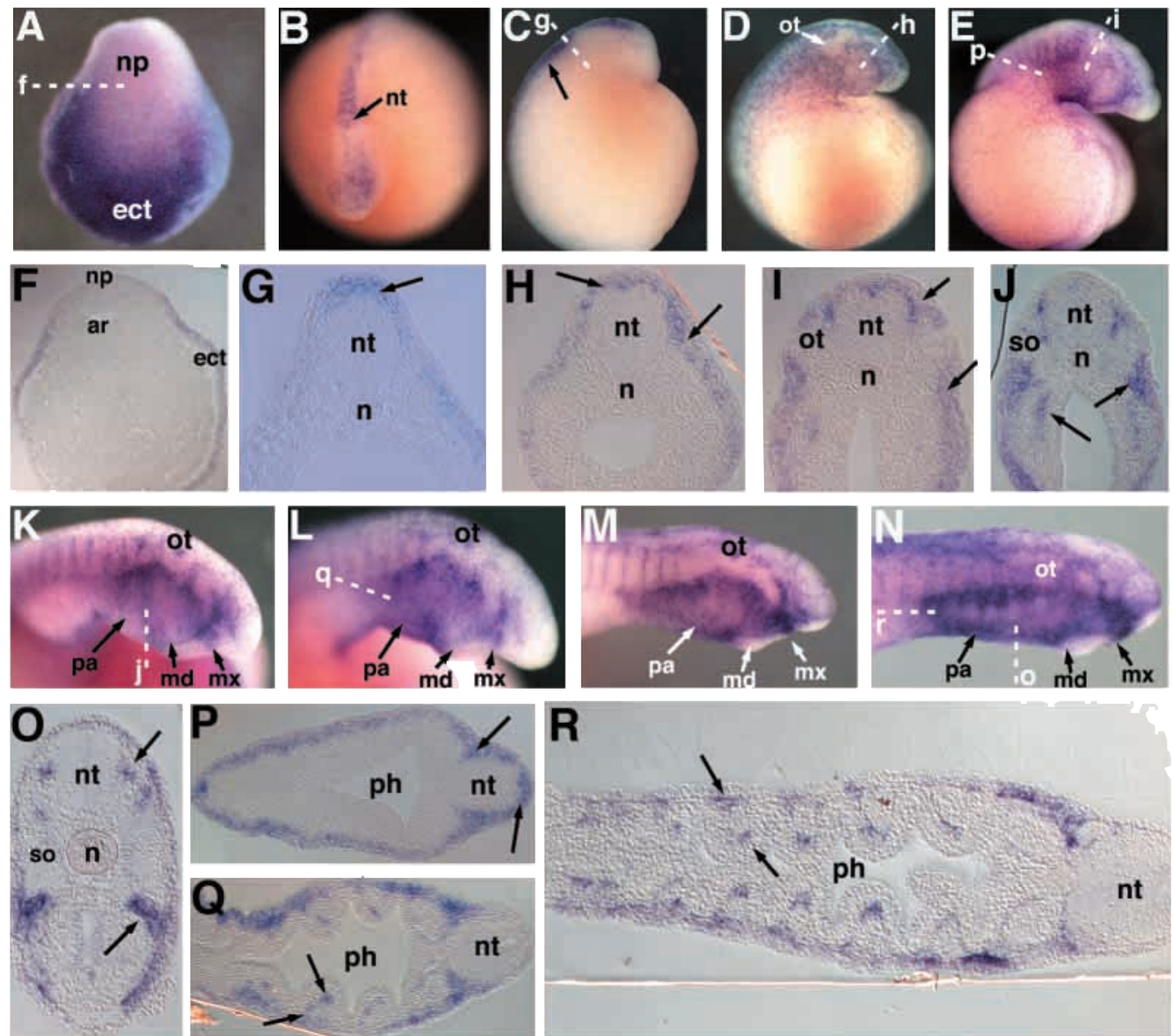
and in mesenchyme above and

below the otic vesicles (arrows). No staining is apparent medial to the vesicle where it approximates the neural tube. (J) Cross-section through 8-day-old embryo in $\mathrm{K}$ at the level of the first somite (j). AP-2 expression is observed in mesenchyme superficial to the ectoderm and adjacent to the gut (arrows). (K) AP-2 expression in the head of an 8-day-old embryo. Staining is most intense in the region of the forming pharyngeal arches. (L) AP-2 expression in the head at 8.5 days, anterior is towards the right. AP-2-positive cells are localized more ventrally than at 8 days. (M) AP-2 expression in the head at 9 days, showing extensive staining in the pharyngeal region. (N) Side view of 11-day-old embryo showing AP-2 expression in the pharyngeal arches and strong staining in the anterior lip. (O) Cross-section through the 11-day-old embryo in $\mathrm{N}$ at position o. AP-2 mRNA is detected in a cluster of neurons in the lateral neural tube and in mesenchyme surrounding the pharynx (arrows). (P) Horizontal section through a 7.5-day-old embryo at the approximate level of $\mathrm{p}$ in $\mathrm{E}$, showing expression in the dorsal neural tube, mesenchyme and ectoderm (arrows). (Q) Horizontal section through the head of the embryo in L at position q. Anterior is towards the right. AP-2-expressing cells are seen in the mesenchyme surrounding the pharyngeal mesoderm (arrows). (R) Horizontal section through an 11-day-old embryo at about the level of $\mathrm{r}$ in $\mathrm{N}$. Expression is observed in the mesenchyme of the pharynx (arrows). ar, archenteron; np, neural plate; ect, non-neural ectoderm; n, notochord; ot, otic pit/vesicle; nt, neural tube/rod; ph, pharynx; pa, pharyngeal arches; md, mandibular arch; mx, maxillary region (anterior lip); so, somite.

ectoderm at open neural plate stages (Fig. 5A). Similar early ectodermal expression has been reported for chick and mouse (Shen et al., 1997; Mitchell et al., 1991). These data suggest an ancient role for AP-2 in the chordate non-neural ectoderm, and strong conservation of an early ectodermal regulatory element in the AP-2 promoter. Interestingly, Dlx and BMP-4 are also co-expressed in the non-neural ectoderm of gnathostomes and amphioxus (Panopoulou et al., 1998; Holland et al., 1996b), and a regulatory relationship between the two has been proposed in frog (Feledy et al., 1999). It is possible that all three genes interact in an evolutionarily ancient pathway for specification of non-neural ectoderm in chordates.

\section{Neural tube expression differs between amphioxus and vertebrates}

Before neural tube formation in vertebrates, AP-2 expression at the neural/non-neural interface increases (Mitchell et al., 1991; Chazaud et al., 1996; Moser et al., 1997; Shen et al., 1997). Simultaneously, expression in the remaining non-neural ectoderm begins to fade. By the completion of neurulation, AP2-positive cells have become incorporated into the dorsal neural tube and epidermal staining is reduced. AP-2 expression in the neural folds and neural tube at these stages mirrors that of neural crest markers Snail, Slug and Id-2, and overlaps with the cranial neural crest markers Dlx-2, Msx-1 and Msx-2 (Robinson and Mahon, 1994; Martinsen and Bronner-Fraser, 1998; Sefton et al., 1998; Bendall and Abate-Shen, 2000).

During parallel stages in amphioxus, the non-neural ectoderm has closed over the forming neural tube and AP-2 transcripts are detected strongly throughout the epidermis. After neurulation, AP-2 is downregulated in the epidermis, but no AP-2-positive cells become incorporated into the dorsal 
neural tube. Subsequent AP-2 expression includes only a few cells in the ventrolateral cerebral vesicle and preoral pit.
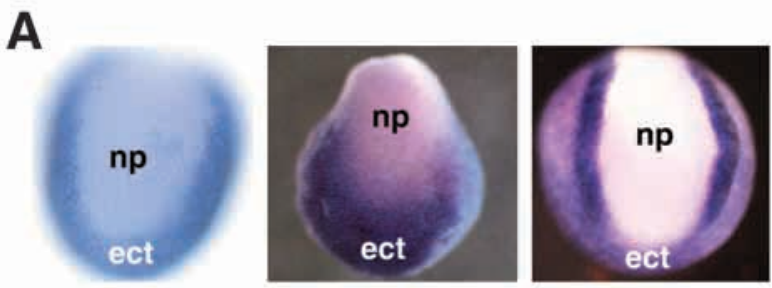

B

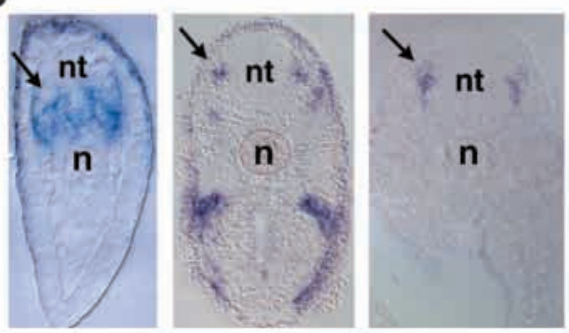

Fig. 5. Conserved aspects of AP-2 expression in amphioxus, lamprey, and gnathostomes. (A) Dorsal anterior view of similarly staged amphioxus (left panel), lamprey (middle panel) and axolotl (right panel) neurulae. In all three embryos, AP-2 is upregulated in the non-neural ectoderm and excluded from the neural plate.

(B) Cross-sections through the anterior neural tube of amphioxus (left panel), lamprey (middle panel) and axolotl (right panel). All three embryos show AP-2 staining in the lateral walls of anterior neural tube (arrows) after neurulation. n, notocord.; np, neural plate; ect, non-neural ectoderm; nt, neural tube.
During these stages, the amphioxus homologs of Snail and Msx are expressed at the edges of the neural plate and then expand throughout the neural tube (Langeland et al., 1997; Sharman et al., 1999). Unlike vertebrates, neither AmphiSnail or AmphiMsx gene expression overlaps with AP-2 in the dorsal neural tube.

Differences in amphioxus and vertebrate AP-2 neural expression imply divergent modes of AP-2 regulation in the two subphyla. A simplistic explanation is the presence of a neural crest enhancer in vertebrate AP-2 gene promoters that is absent in the homologous amphioxus promoter. Candidate regulators for a putative novel enhancer would include genes such as Snail and Msx, which are co-expressed with AP-2 in vertebrate neural crest, but not in the amphioxus neural tube. Provocatively, the murine Msx-1 promoter contains a consensus AP-2-binding site (Kuzuoka et al., 1994). Thus, it is possible that new regulatory relationships between these genes resulted in novel deployment of AP-2 to neural crest early in vertebrate evolution. An alternative explanation is novel deployment of the upstream regulators of AP-2 in vertebrates. Furthermore, secondary loss of a neural enhancer, or differential deployment of trans-acting regulators may have resulted in a loss of AP-2 expression in the amphioxus dorsal neural tube. Expression data from the third chordate subphylum, Urochordata, may clarify the direction of this evolutionary change.

Given its essential role in cranial neural crest cell differentiation and proliferation, it is tempting to speculate that co-option of AP-2 by the dorsal neural tube was a crucial event in neural crest evolution. Knockout studies of AP- $2 \alpha$ demonstrate the necessity of AP-2 activity in post-migratory
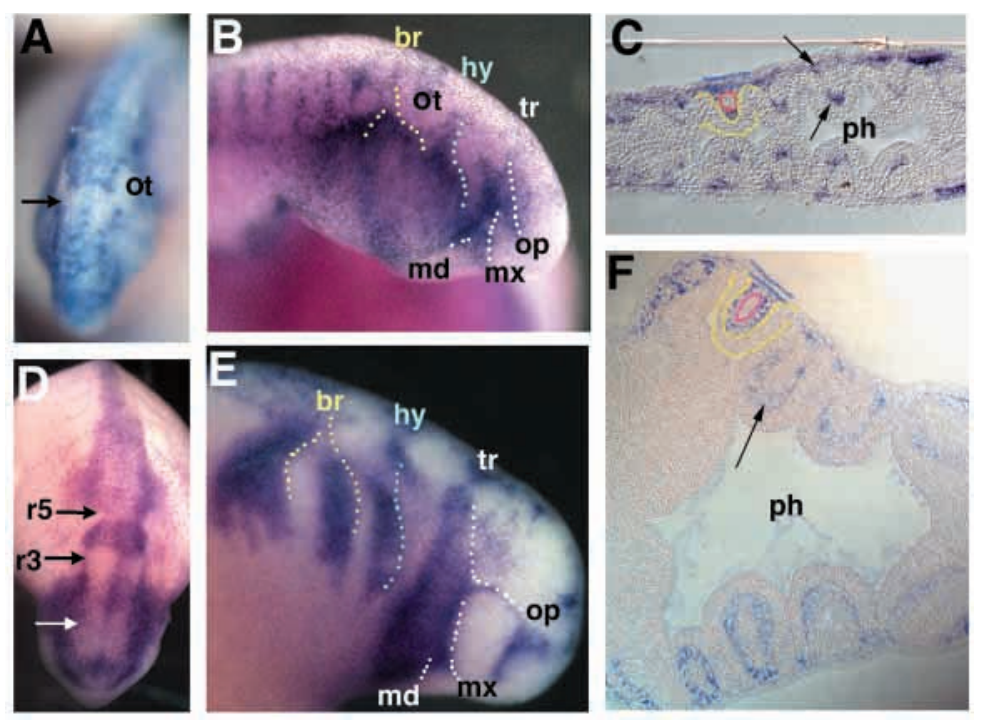

(black arrows) and a region of rhombomere 1 (white arrow). Compared with the lamprey embryo in A, axolotl shows greater patterning of AP-2 expression along the anterior neural tube. (E) Side view of approximately stage 25 axolotl embryo showing AP-2 expression in cranial neural crest streams. Streams are marked with dotted lines as in B. White dots border the trigeminal stream, which splits into the ophthalmic, maxillary and mandibular streams. Blue dots border the hyoid stream, and yellow dots border the first branchial and common 2/3/4 branchial streams. Overall, the distribution of AP-2 positive cells in the axolotl head resembles that of lamprey (B), except ventrally, where the divisions between putative neural crest streams are much less defined in lamprey than in axolotl. (F) Horizontal sections through the pharynx of stage $\sim 30$ axolotl embryo. Germ layers are marked as in C. AP-2-positive cells are observed in the mesenchyme surrounding the arch mesoderm (arrow). Both lamprey (C) and axolotl have AP-2-positive cells lying medial to the pharyngeal arch mesoderm. ot, otic pit/vesicle; md, mandibular neural crest stream; mx, maxillary neural crest stream; op, ophthalmic neural crest stream; br, branchial neural crest stream; hy, hyoid neural crest stream; tr, trigeminal neural crest stream; ph, pharynx; r3, rhombomere 3; r5, rhombomere 5. 
cranial neural crest (Morriss-Kay, 1996; Schorle et al., 1996; Zhang et al., 1996). Mice that lack AP-2 have relatively normal neural crest migration, but most neural crest derivatives in the head (including the rostral-most parts of the skull, the first and second arch cartilages, and cranial sensory ganglia) are missing or reduced. Thus, AP-2 expression in the dorsal neural tube may have been a prerequisite for the evolution of neural crest cells

If AP-2 use in the dorsal neural tube is indeed a vertebrate apomorphy, an intriguing issue is whether its new roles in neural crest involved evolution of the AP-2 protein itself, or simply redeployment of a functionally conserved gene. Although all described AP-2 protein sequences are highly conserved in the DNA-binding/dimerization domains, novel motifs in the more divergent transactivation domain may confer additional regulatory properties onto vertebrate AP-2 genes. In vivo and in vitro comparisons of amphioxus and lamprey AP-2 gene function may shed light on the biochemical features important for AP-2 function in the neural crest.

\section{Later AP-2 expression in amphioxus}

Shortly after expression has faded from the epidermis in amphioxus, AP-2 is upregulated in cells of the ventrolateral cerebral vesicle and forming preoral pit. In axolotl and lamprey, AP-2 has a potentially homologous late phase of expression in neurons of the anterior neural tube (Fig. 5B). AP2 expression in the developing cerebellum of mouse also has been reported (Moser et al., 1997). Furthermore, AP-2 expression in the developing fly brain is a prominent feature of Drosophila AP-2 expression (Monge and Mitchell, 1998), suggesting an ancient function for AP-2 genes in the anterior nervous system of bilaterians.

Enrichment in the developing pre-oral pit is harder to relate to any aspect of vertebrate expression. It may reflect, however, an evolutionarily conserved regulatory relationship between AP-2 and Hox 2 genes. AmphiHox 2 expression temporally and spatially overlaps with AP-2 in the preoral pit (Wada et al., 1999), and AP-2 genes are essential for HoxA2 expression in cranial neural crest (Maconochie et al., 1999). Amphioxus AP2 may similarly regulate Hox 2 in the preoral pit, as two consensus AP-2 binding sites are present in the $5^{\prime}$ genomic sequence of AmphiHox2. AP-2 expression in Drosophila also overlaps with proboscipedia (Hox2) (Monge and Mitchell, 1998). Three consensus AP-2 binding sites are found clustered within a $1 \mathrm{~kb}$ intronic region shown to direct reporter expression to the maxillary lobe. Taken together, these observations suggest an ancient role for AP-2 in Hox class 2 gene regulation.

\section{AP-2 and neural crest migration patterns in lamprey}

Our results show that AP-2 expression in lamprey closely resembles AP-2 expression in axolotl, chicken and mouse. Early deployment in ectoderm is followed by expression in the neural folds and dorsal neural tube. AP-2 transcripts are then seen throughout the head mesenchyme, in a pattern consistent with expression in early migrating neural crest. Later, AP-2 staining in the head is confined to apparent streams or blocks of cells. Finally, lamprey AP-2 appears in mesenchyme surrounding pharyngeal arch mesoderm. The similarity of lamprey AP-2 and gnathostome AP-2 staining, together with the anatomical context of lamprey AP-2 expression, strongly suggests that AP-2 marks neural crest cells in lamprey.

Using AP-2 as a marker gives valuable insight into the migration patterns of neural crest cells in a basal vertebrate. Previous studies have analyzed lamprey neural crest migration using scanning electron microscopy, molecular markers for subsets of crest cells (Otx, Dlx) (Tomsa and Langeland, 1999; Neidert et al., 2001) or limited DiI labeling (Horigome et al., 1999). The current study is the first time expression of a pan-neural crest marker has been analyzed in lamprey. Comparing lamprey and axolotl AP-2 expression patterns illustrates that lamprey cranial neural crest migrates in typical vertebrate fashion. Three broad areas of AP-2 expression can be discerned in the lamprey head, which appear equivalent to the trigeminal, hyoid and branchial streams in gnathostomes (Fig. 6B,E). Furthermore, the hyoid and branchial streams appear to lie on either side of the otic vesicle, as in gnathostomes. This contradicts previous scanning electron microscopy analyses suggesting that lamprey hyoid neural crest migrates directly under the otic vesicle (Horigome et al., 1999). In sections through lamprey embryos, no AP-2-positive cells are observed interior to the otic vesicle (Fig. 4I).

Interestingly, during the early stages of neural crest migration (6-7 days), a gap in AP-2 expression appears in the neural tube just anterior to the otic placode (Fig. 6A). In gnathostomes, similar gaps correspond to rhombomeres 3 and 5, which are depleted of neural crest (Fig. 6D). We cannot be sure if this gap corresponds to a rhombomere as no molecular or anatomical rhombomeric markers are available for this stage in lamprey. However, the presence of only one gap is suggestive of reduced patterning in the early migrating neural crest of lamprey.

At later stages, the putative trigeminal stream appears to divide into the ophthalmic stream rostrally, and 'maxillomandibular' caudally (Fig. 6B). The maxillo-mandibular then splits around the mouth, filling the maxillary (anterior lip) and mandibular regions. This subdivision mimics streaming patterns in gnathostomes as illustrated by AP-2 staining in axolotl (Fig. 6E) and supports homology of lamprey and gnathostome mandibular segments. This finding, along with recent studies of engrailed and Otx expression, lend molecular support to the idea that gnathostome jaws evolved from the pumping organ of an agnathan ancestor, rather than anterior gill arch cartilage.

Although initial subdivision of putative neural crest cells in lamprey closely mimics that of gnathostomes, later ventral migration into the nascent pharyngeal arches is somewhat different. Conspicuously, coherent streaming of lamprey cranial neural crest is not maintained as the cells move ventrally, and the three streams appear to fuse as they fill the pharyngeal region (compare Fig. 6B with 6E). Subsequent partitioning of pharyngeal arch neural crest appears to occur only after migration as the outpocketing endoderm divides both the paraxial mesoderm and overlying neural crest (Fig. 4N). This difference in streaming pattern may reflect a heterochrony in arch formation relative to neural crest migration between lamprey and gnathostomes.

A long-recognized difference between lamprey and gnathostome cranial neural crest is its final destination in the arches (Graham, 2001; Kimmel et al., 2001). In gnathostomes, 
cartilages derived from cranial neural crest lie medial to the arch mesoderm. In lamprey, this support tissue lies lateral to the arch mesoderm. This is reflected by AP-2 staining in axolotl, showing neural crest cells internal to the arch mesoderm (Fig. 6F). We find that in lamprey, AP-2 transcripts are similarly distributed internal to the pharyngeal arch mesoderm (Fig. 6C), suggesting medial movement of pharyngeal arch neural crest does indeed occur in lamprey, although to a lesser degree than in gnathostomes.

\section{Conclusions}

In this study, we have documented differences in AP-2 regulation across the evolutionary transitions from invertebrate to vertebrate and agnathan to gnathostome. AP-2 expression in amphioxus and vertebrates implies co-option of AP-2 by neural crest cells in the vertebrate lineage. This was a potentially crucial event in vertebrate evolution, as AP-2 has essential roles in cranial neural crest differentiation and proliferation. AP-2 deployment in the neural tube may have potentiated neural crest evolution by promoting transcription of downstream effectors of cranial neural crest differentiation. AP-2 expression patterns in lamprey and axolotl demonstrate an increase in neural crest patterning in gnathostomes, and elaboration of neural crest migratory behavior that may relate to the timing of pharyngeal arch formation. As in situ hybridization is merely a series of static observations of the use of a single gene, conclusive proof of these differences await the results of detailed cell tracking experiments. Taken together, the regulatory history of AP-2 genes in the chordate lineage suggest molecular and developmental mechanisms for the evolution of the vertebrate head.

We thank Linda and Nick Holland for making amphioxus collection possible, John Lawrence for laboratory space in Tampa, Jim Langeland for generously providing excellent cDNA libraries, David McCauley for supplying lamprey embryos, and Robert Cerny for invaluable discussion. This work was supported by NASA grant 98HEDS-02 to M. B.-F.

\section{REFERENCES}

Bauer, R., Imhof, A., Pscherer, A., Kopp, H., Moser, M., Seegers, S., Kerscher, M., Tainsky, M. A., Hofstaedter, F. and Buettner, R. (1994). The genomic structure of the human Ap-2 transcription factor. Nucleic Acids Res. 22, 1413-1420.

Bauer, R., McGuffin, M. E., Mattox, W. and Tainsky, M. A. (1998). Cloning and characterization of the Drosophila homologue of the AP-2 transcription factor. Oncogene 17, 1911-1922.

Bendall, A. J. and Abate-Shen, C. (2000). Roles for Msx and Dlx homeoproteins in vertebrate development. Gene 247, 17-31.

Chazaud, C., OuladAbdelghani, M., Bouillet, P., Decimo, D., Chambon, P. and Dolle, P. (1996). AP-2.2, a novel gene related to AP-2, is expressed in the forebrain, limbs and face during mouse embryogenesis. Mech. Dev. 54, 83-94.

Epperlein, H. H., Meulemans, D., Bronner-Fraser, M., Steinbeisser, H. and Selleck, M. A. J. (2000). Analysis of cranial neural crest migratory pathways in axolotl using cell markers and transplantation. Development 127, 2751-2761.

Feledy, J. A., Beanan, M. J., Sandoval, J. J., Goodrich, J. S., Lim, J. H., Matsuo-Takasaki, M., Sato, S. M. and Sargent, T. D. (1999). Inhibitory patterning of the anterior neural plate in Xenopus by homeodomain factors Dlx3 and Msx1. Dev. Biol. 212, 455-464.

Force, A., Amores, A. and Postlethwait, J. H. (2002). Hox cluster organization in the jawless vertebrate Petromyzon marinus. J. Exp. Zool. 294, 30-46.
Gans, C. and Northcutt, R. G. (1983). Neural crest and the origin of vertebrates - a new head. Science 220, 268-274.

Graham, A. (2001). The development and evolution of the pharyngeal arches. J. Anat. 199, 133-141.

Henrique, D., Adam, J., Myat, A., Chitnis, A., Lewis, J. and Ishhorowicz, D. (1995). Expression of a Delta-homolog in prospective neurons in the chick. Nature 375, 787-790.

Hilger-Eversheim, K., Moser, M., Schorle, H. and Buettner, R. (2000). Regulatory roles of AP-2 transcription factors in vertebrate development, apoptosis and cell-cycle control. Gene 260, 1-12.

Holland, L. Z. and Holland, N. D. (2001). Evolution of neural crest and placodes: amphioxus as a model for the ancestral vertebrate? J. Anat. 199, 85-98.

Holland, L. Z., Holland, P. W. H. and Holland, N. D. (1996a). Revealing homologies between body parts of distantly related animals by in situ hybridization to developmental genes: amphioxus versus vertebrates. In Molecular Zoology: Advances, Strategies, and Protocols (ed. J. D. Ferraris and S. R. Palumbi), pp. 267-282, 473-483. New York: Wiley.

Holland, N. D., Panganiban, G., Henyey, E. L. and Holland, L. Z. (1996b). Sequence and developmental expression of AmphiDII, an amphioxus Distalless gene transcribed in the ectoderm, epidermis and nervous system: Insights into evolution of craniate forebrain and neural crest. Development 122, 2911-2920.

Holland, P. W. H. (1999). Gene duplication: past, present and future. Semin. Cell Dev. Biol. 10, 541-547.

Horigome, N., Myojin, M., Ueki, T., Hirano, S., Aizawa, S. and Kuratani, S. (1999). Development of cephalic neural crest cells in embryos of Lampetra japonica, with special reference to the evolution of the law. Dev. Biol. 207, 287-308.

Kimmel, C. B., Miller, C. T. and Keynes, R. J. (2001). Neural crest patterning and the evolution of the jaw. J. Anat. 199, 105-120.

Kuzuoka, M., Takahashi, T., Guron, C. and Raghow, R. (1994). Murine homeobox-containing gene, Msx-1 - analysis of genomic organization, promoter structure, and potential autoregulatory cis-acting elements. Genomics 21, 85-91.

LaBonne, C. and Bronner-Fraser, M. (1999). Molecular mechanisms of neural crest formation. Ann. Rev. Cell Dev. Biol. 15, 81-112.

Langeland, J. A., Tomsa, J. M., Jackman, W. R. and Kimmel, C. B. (1997). AmphiSnail: structure, phylogeny, and developmental expression of an amphioxus snail homolog. Dev. Biol. 186, A28-A28.

Langille, R. M. and Hall, B. K. (1988). Role of the neural crest in development of the trabeculae and branchial arches in embryonic sea lamprey, Petromyzon-marinus (L). Development 102, 301-310.

Maconochie, M., Krishnamurthy, R., Nonchev, S., Meier, P., Manzanares, M., Mitchell, P. J. and Krumlauf, R. (1999). Regulation of Hoxa2 in cranial neural crest cells involves members of the AP-2 family. Development 126, 1483-1494.

Martinsen, B. J. and Bronner-Fraser, M. (1998). Neural crest specification regulated by the helix-loop-helix repressor Id2. Science 281, 988-991.

Mitchell, P. J., Timmons, P. M., Hebert, J. M., Rigby, P. W. J. and Tjian, R. (1991). Transcription Factor Ap-2 Is Expressed in Neural Crest Cell Lineages During Mouse Embryogenesis. Genes Dev. 5, 105-119.

Monge, I. and Mitchell, P. J. (1998). DAP-2, the Drosophila homolog of transcription factor AP-2. Mech. Dev. 76, 191-195.

Morriss-Kay, G. M. (1996). Craniofacial defects in AP-2 null mutant mice. BioEssays 18, 785-788.

Moser, M., Ruschoff, J. and Buettner, R. (1997). Comparative analysis of AP-2 alpha and AP-2 beta gene expression during murine embryogenesis. Dev. Dyn. 208, 115-124.

Myojin, M., Ueki, T., Sugahara, F., Murakami, Y., Shigetani, Y., Aizawa, S., Hirano, S. and Kuratani, S. (2001). Isolation of Dlx and Emx gene cognates in an agnathan species, Lampetra japonica, and their expression patterns during embryonic and larval development: conserved and diversified regulatory patterns of homeobox genes in vertebrate head evolution. J. Exp. Zool. 291, 68-84.

Neidert, A. H., Virupannavar, V., Hooker, G. W. and Langeland, J. A. (2001). Lamprey Dlx genes and early vertebrate evolution. Proc. Natl. Acad. Sci. USA 98, 1665-1670.

Ogasawara, M., Shigetani, Y., Hirano, S., Satoh, N. and Kuratani, S. (2000). Pax1/Pax9-related genes in an agnathan vertebrate, Lampetra japonica: expression pattern of LjPax9 implies sequential evolutionary events toward the gnathostome body plan. Dev. Biol. 223, 399-410.

Panopoulou, G. D., Clark, M. D., Holland, L. Z., Lehrach, H. and Holland, N. D. (1998). AmphiBMP2/4, an amphioxus bone morphogenetic protein 
closely related to Drosophila decapentaplegic and vertebrate BMP2 and BMP4: Insights into evolution of dorsoventral axis specification. Dev. Dyn. 213, 130-139.

Robinson, G. W. and Mahon, K. A. (1994). Differential and overlapping expression domains of Dlx-2 and Dlx-3 suggest distinct roles for Distal-less homeobox genes in craniofacial development. Mech. Dev. 48, 199-215.

Saitou, N. and Nei, M. (1987). The neighbor-joining method - a new method for reconstructing phylogenetic trees. Mol. Biol. Evol. 4, 406-425.

Sasai, N., Mizuseki, K. and Sasai, Y. (2001). Requirement of FoxD3-class signaling for neural crest determination in Xenopus. Development 128, 2525-2536.

Schorle, H., Meier, P., Buchert, M., Jaenisch, R. and Mitchell, P. J. (1996) Transcription factor AP-2 essential for cranial closure and craniofacial development. Nature 381, 235-238.

Sefton, M., Sanchez, S. and Nieto, M. A. (1998). Conserved and divergent roles for members of the Snail family of transcription factors in the chick and mouse embryo. Development 125, 3111-3121.

Sharman, A. C. and Holland, P. W. H. (1998). Estimation of Hox gene cluster number in lampreys. Int. J. Dev. Biol. 42, 617-620.

Sharman, A. C., Shimeld, S. M. and Holland, P. W. H. (1999). An amphioxus Msx gene expressed predominantly in the dorsal neural tube. Dev. Genes Evol. 209, 260-263.

Shen, H., Wilke, T., Ashique, A. M., Narvey, M., Zerucha, T., Savino, E.,
Williams, T. and Richman, J. M. (1997). Chicken transcription factor AP2: cloning, expression and its role in outgrowth of facial prominences and limb buds. Dev. Biol. 188, 248-266.

Thompson, J. D., Gibson, T. J., Plewniak, F., Jeanmougin, F. and Higgins, D. G. (1997). The CLUSTAL_X windows interface: flexible strategies for multiple sequence alignment aided by quality analysis tools. Nucleic Acids Res. 25, 4876-4882.

Tomsa, J. M. and Langeland, J. A. (1999). Otx expression during lamprey embryogenesis provides insights into the evolution of the vertebrate head and jaw. Dev. Biol. 207, 26-37.

Ueki, T., Kuratani, S., Hirano, S. and Aizawa, S. (1998). Otx cognates in a lamprey, Lampetra japonica. Dev. Genes Evol. 208, 223-228.

Wada, H., Garcia-Fernandez, J. and Holland, P. W. H. (1999). Colinear and segmental expression of amphioxus Hox genes. Dev. Biol. 213, 131141.

Zhang, J. A., HagopianDonaldson, S., Serbedzija, G., Elsemore, J., PlehnDujowich, D., McMahon, A. P., Flavell, R. A. and Williams, T. (1996). Neural tube, skeletal and body wall defects in mice lacking transcription factor AP-2. Nature 381, 238-241.

Zhao, F., Satoda, M., Licht, J. D., Hayashizaki, Y. and Gelb, B. D. (2001). Cloning and characterization of a novel mouse AP-2 transcription factor, Ap-2 gamma, with unique DNA binding and transactivation properties. $J$. Biol. Chem. 276, 40755-40760 